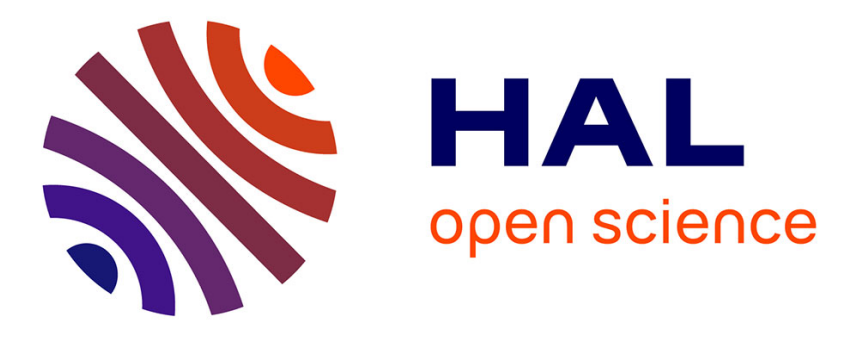

\title{
Tracking improvement based on the Proxy control scheme for bilateral teleoperation system under time-varying delays
}

Bo Zhang, Alexandre Kruszewski, Jean-Pierre Richard

\section{- To cite this version:}

Bo Zhang, Alexandre Kruszewski, Jean-Pierre Richard. Tracking improvement based on the Proxy control scheme for bilateral teleoperation system under time-varying delays. 16th IEEE International Conference on Emerging Technologies and Factory Automation (ETFA), Sep 2011, Toulouse, France. hal-00602337

\section{HAL Id: hal-00602337 \\ https://hal.science/hal-00602337}

Submitted on 22 Jun 2011

HAL is a multi-disciplinary open access archive for the deposit and dissemination of scientific research documents, whether they are published or not. The documents may come from teaching and research institutions in France or abroad, or from public or private research centers.
L'archive ouverte pluridisciplinaire HAL, est destinée au dépôt et à la diffusion de documents scientifiques de niveau recherche, publiés ou non, émanant des établissements d'enseignement et de recherche français ou étrangers, des laboratoires publics ou privés. 


\title{
Tracking Improvement Based on the Proxy Control Scheme for Bilateral Teleoperation System under Time-Varying Delays
}

\author{
Bo Zhang, Alexandre Kruszewski, Jean-Pierre Richard ${ }^{1}$ \\ LAGIS, CNRS FRE3303, Laboratoire d'Automatique, Génie Informatique et Signal \\ Université Lille Nord de France, Ecole Centrale de Lille, 59651 Villeneuve d'Ascq, France \\ zhangbo829@yahoo.com.cn, \{alexandre.kruszewski, jean-pierre.richard $\} @$ ec-lille.fr
}

\begin{abstract}
This paper addresses the problem of the position/force tracking in teleoperation system and proposes a haptic proxy control scheme. Compared to previous works, communication delays are assumed to be both time-varying and asymmetric, and the response of the synchronization and the transparency are improved. The control design is performed using Linear Matrix Inequality (LMI) optimization based on Lyapunov-Krasovskii functionals (LKF) and $H_{\infty}$ control theory. With the designed controllers, the simulations of different working conditions, such as abrupt motion and wall contact, are performed and show the effectiveness of the proposed solution.
\end{abstract}

\section{Introduction}

A teleoperation system is an extension of human manipulative capabilities to the remote environment. A typical teleoperation system is composed of the human operator, the master haptic-interface robot, the communication medium, the slave robot and the environment. If the position/velocity/force information of the master robot handled by the human operator are only transmitted from the master to the slave, the teleoperation system is called unilateral, and if the corresponding data of the slave is also transmitted back to the master, the teleoperation system is bilateral, we will deal with this second case $[1,12]$.

In bilateral teleoperation, the master and slave are coupled via the communication medium, which incurs the additional dynamics represented by time-varying delays (especially with the internet) $[13,16,22]$. In order to avoid a severe deterioration of the global performance, these delays must be considered at the control design stage [2]. Many methods have been presented to address the stability problems with time delays, for which the the passivity formalism based on the Velocity-Force (VF) scheme is a prevalent one. Since the cornerstone papers of Anderson and Spong [1], Niemeyer and Slotine [15], the conception of the passivity, the scattering and the waving variable

\footnotetext{
${ }^{1}$ Jean-Pierre Richard is also with INRIA NON-A.
}

has been extensively studied for the controller design in a passive and stable fashion. Besides, another formulation is the passivity-based structure without the transformation of wave variables. A recent approach is the energy/power based time domain passivity control $[17,21]$. Overall, the latest passivity-based method can deal with the stabilization and the velocity tracking under time-varying delays, but does not allow for optimizing the system performance, especially, does not guarantee the position/force tracking in general.

For the cases of non-passive approach, various control strategies have been proposed. $[8,11,18]$ presented a variety of novel architectures to guarantee the system performance. While these approaches are very inspiring, the time delays are assumed to be constant or symmetric. Recently, some other non-passive based strategies have been proposed, in $[4,7,12,14]$, in order to deal with the timevarying delays, but for these methods, the synchronization or the transparency of the teleoperation system is hard to guarantee.

Apart from the basic stability of teleoperation system under delays, there are primarily two kinds of performance of the bilateral teleoperation system. Firstly, the slave robot should track the position of the master manipulator, and secondly, the environmental force acting on the slave, when it contacts the external environment, should be accurately and real-time transmitted to the master [3]. These two objectives are defined as the position/force tracking, which will be resolved in this paper. With this aim in mind, Lyapunov approaches for timedelay systems are helpful. In the case of time-varying delays, many stability conditions have been proposed in terms of Lyapunov-Krasovskii functionals (LKF), which can be solved by Linear Matrix Inequalities (LMI) [5](see also the included references). For the performance consideration, the $H_{\infty}$ control theory will be used [6, 20, 24]. In short, in this paper, we will propose a novel proxy control scheme for the bilateral teleoperation system under variable and asymmetric delays, which makes use of LKF to ensure the stability, and further, realize the position tracking by $H_{\infty}$ control. Finally, the characteristic of the control scheme can assure the force tracking. 
This paper is organized as follows: Section 2 introduces the theorems to be used later. The problem and the assumptions under consideration are presented in Section 3. In Section 4, the bilateral teleoperation system using the LKF and $H_{\infty}$ control is given. Results of simulation are presented in Section 5. Finally we conclude and discuss the future work in Section 6.

\section{Preliminaries}

This section is devoted to the stability analysis with $H_{\infty}$ performance index. For the simplicity, the teleoperation system will be modeled as a linear time-varying delay system. Firstly, let us consider a bilateral teleoperation system without the time-varying delays, but with the perturbation,

$$
\left(\Sigma_{1}\right) \begin{cases}\dot{x}(t) & =A x(t)+B w(t) \\ z(t) & =C x(t)\end{cases}
$$

Where, $x(t) \in \mathbf{R}^{n}$ is the system state, $w(t) \in \mathbf{R}^{l}$ is defined as the exogenous disturbance signal, and $z(t) \in$ $\mathbf{R}^{q}$ is seen as the objective control output, $A, B$ and $C$ are constant matrices.

For ensuring the stability of system and guaranteeing the improvement of the overall performance, Bounded Real Lemma (BRL) with Lyapunov functional stability condition is used to derive LMI stability conditions [6].

With a prescribed scalar $\gamma$, we define the performance index,

$$
J(w)=\int_{0}^{\infty}\left(z(t)^{T} z(t)-\gamma^{2} w(t)^{T} w(t)\right) d t
$$

Then, according to the theory of $H_{\infty}$ control, we can ensure the stability and optimize the performance of the system by verifying the performance index,

$$
J(w)<0
$$

So, we obtain the following theorem with the consideration of $H_{\infty}$ control [6],

Theorem 1 Suppose there exists $n \times n$ matrices $P>0$, $P_{2}, P_{3}$, and a positive scale $\gamma$, such that the condition (4) with the notation (5) is feasible, the system (1) is asymptotically stable and $J(w)<0$.

$$
\begin{gathered}
\Gamma^{1}=\left(\begin{array}{ccc}
\Gamma_{11}^{1} & P-P_{2}^{T}+A^{T} P_{3} & P_{2}^{T} B \\
* & -P_{3}-P_{3}^{T} & P_{3}^{T} B \\
* & * & -\gamma^{2} I
\end{array}\right)<0 \\
\Gamma_{11}^{1}=A^{T} P_{2}+P_{2}^{T} A+C^{T} C
\end{gathered}
$$

Based on the theorem 1, we consider the bilateral teleoperation system with the time-varying delays and the perturbation, the system is generally modeled as follow,
$\left(\Sigma_{2}\right)\left\{\begin{array}{l}\dot{x}(t)=\sum_{i=0}^{n} A_{i} x\left(t-\tau_{i}(t)\right)+B w(t) \\ z(t)=C x(t) \\ x\left(t_{0}+\theta\right)=\phi(\theta), \dot{x}\left(t_{0}+\theta\right)=\dot{\phi}(\theta), \theta \in\left[-h_{2}, 0\right]\end{array}\right.$

Where, $\tau_{0}(t) \equiv 0, \phi(\theta)$ is the initial condition, and the time-varying delays, $\tau_{i}(t) \in\left[h_{1}, h_{2}\right], h_{1} \geq 0$, $i=1,2, \ldots, n$. Considering the following LyapunovKrasovskii functional [5],

$$
\begin{aligned}
& V(t, x(t), \dot{x}(t))=x(t)^{T} P x(t) \\
& +\int_{t-h_{2}}^{t} x(s)^{T} S_{a} x(s) d s+\int_{t-h_{1}}^{t} x(s)^{T} S x(s) d s \\
& +h_{1} \int_{-h_{1}}^{0} \int_{t+\theta}^{t} \dot{x}(s)^{T} R \dot{x}(s) d s d \theta \\
& +\sum_{i=1}^{n}\left(h_{2}-h_{1}\right) \int_{-h_{2}}^{-h_{1}} \int_{t+\theta}^{t} \dot{x}(s)^{T} R_{a i} \dot{x}(s) d s d \theta
\end{aligned}
$$

Theorem 2 [23] Suppose there exists $n \times n$ matrices $P>0, R>0, S>0, S_{a}>0, R_{a i}>0, P_{2}, P_{3}, Y_{1}, Y_{2}$, $i=1,2, \ldots, n$, and a positive scale $\gamma$, such that the condition (8) with the notation (9) is feasible, the system (6) is asymptotically stable and $J(w)<0$ for time-varying delays $\tau_{i}(t) \in\left[h_{1}, h_{2}\right], i=1,2, \ldots, n$.

Proof. The proof can be found in the theorem 2 of [23]. To ensure $J(w)<0$, the term $z(t)^{T} z(t)-\gamma^{2} w(t)^{T} w(t)$ can be added into $\dot{V}(t, x(t), \dot{x}(t))$. Considering the system of (6), we get,

$$
\begin{aligned}
& \dot{V}(t, x(t), \dot{x}(t))+z(t)^{T} z(t)-\gamma^{2} w(t)^{T} w(t) \\
& =x(t)^{T}\left(S+S_{a}\right) x(t) \\
& +\dot{x}(t)^{T} P x(t)+x(t)^{T} P \dot{x}(t) \\
& -x\left(t-h_{1}\right)^{T} S x\left(t-h_{1}\right) \\
& -x\left(t-h_{2}\right)^{T} S_{a} x\left(t-h_{2}\right) \\
& +\dot{x}(t)^{T}\left[h_{1}^{2} R+\left(h_{2}-h_{1}\right)^{2} \sum_{i=1}^{n} R_{a i}\right] \dot{x}(t) \\
& -h_{1} \int_{t-h_{1}}^{t} \dot{x}(s)^{T} R \dot{x}(s) d s \\
& -\left(h_{2}-h_{1}\right) \int_{t-h_{2}}^{t-h_{1}} \dot{x}(s)^{T} \sum_{i=1}^{n} R_{a i} \dot{x}(s) d s \\
& +z(t)^{T} z(t)-\gamma^{2} w(t)^{T} w(t)
\end{aligned}
$$

Then, substituting for $z(t)$, applying the Jensen's inequality [9], then obtain, 
$\Gamma^{2}=\left(\begin{array}{ccc}\Gamma_{11}^{2} & \Gamma_{12}^{2} & R+\sum_{i=1}^{n} P_{2}^{T} A_{i}-n Y_{1}^{T} \\ * & \Gamma_{22}^{2} & \sum_{i=1}^{n} P_{3}^{T} A_{i}-n Y_{2}^{T} \\ * & * & -S-R \\ * & * & * \\ * & * & * \\ * & * & * \\ * & * & * \\ * & * & * \\ * & * & * \\ * & * & * \\ * & * & *\end{array}\right.$
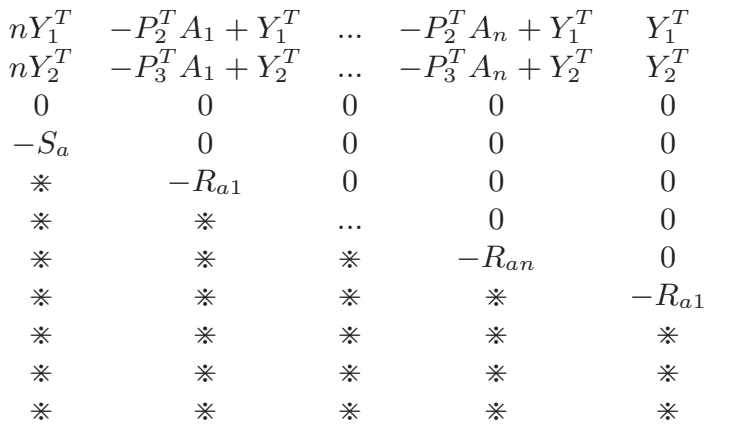

$\begin{array}{cc}\cdots & Y_{1}^{T} \\ \cdots & Y_{2}^{T} \\ 0 & 0 \\ 0 & 0 \\ 0 & 0 \\ 0 & 0 \\ 0 & 0 \\ 0 & 0 \\ \cdots & 0 \\ * & -R_{a n} \\ * & *\end{array}$

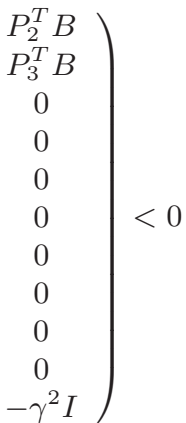

(8)

$\Gamma_{11}^{2}=S+S_{a}-R+A_{0}^{T} P_{2}+P_{2}^{T} A_{0}+C^{T} C, \quad \Gamma_{12}^{2}=P-P_{2}^{T}+A_{0}^{T} P_{3}, \quad \Gamma_{22}^{2}=-P_{3}-P_{3}^{T}+h_{1}^{2} R+\left(h_{2}-h_{1}\right)^{2} \sum_{i=1}^{n} R_{a i}$

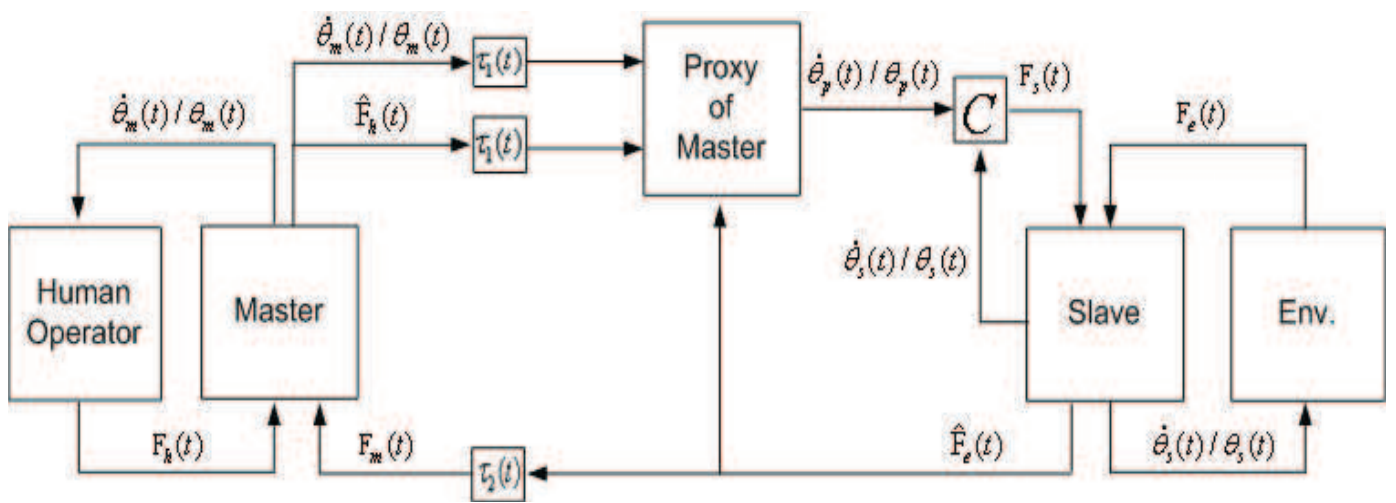

Figure 1. Novel proxy control scheme.

$$
\begin{aligned}
& \dot{V}(t, x(t), \dot{x}(t))+z(t)^{T} z(t)-\gamma^{2} w(t)^{T} w(t) \\
& \leq x(t)^{T}\left(S+S_{a}+C^{T} C\right) x(t) \\
& +\dot{x}(t)^{T} P x(t)+x(t)^{T} P \dot{x}(t) \\
& -x\left(t-h_{1}\right)^{T} S x\left(t-h_{1}\right)-x\left(t-h_{2}\right)^{T} S_{a} x\left(t-h_{2}\right) \\
& +\dot{x}(t)^{T}\left[h_{1}^{2} R+\left(h_{2}-h_{1}\right)^{2} \sum_{i=1}^{n} R_{a i}\right] \dot{x}(t) \\
& -\left[x(t)^{T}-x\left(t-h_{1}\right)^{T}\right] R\left[x(t)-x\left(t-h_{1}\right)\right] \\
& -\sum_{i=1}^{n} v_{1 i}^{T} R_{a i} v_{1 i}-\sum_{i=1}^{n} v_{2 i}^{T} R_{a i} v_{2 i} \\
& -w(t)^{T} \gamma^{2} I w(t)
\end{aligned}
$$

where,

$$
\begin{aligned}
v_{1 i} & =\int_{t-\tau_{i}(t)}^{t-h_{1}} \dot{x}(s) d s \\
v_{2 i} & =\int_{t-h_{2}}^{t-\tau_{i}(t)} \dot{x}(s) d s, \quad i=1,2, \ldots, n
\end{aligned}
$$

By using the descriptor method and free weighting matrices [5, 10], for some $n \times n$ matrices $P_{2}, P_{3}, Y_{1}, Y_{2}$, the expression as follows is added into $\dot{V}(t, x(t), \dot{x}(t))+$ $z(t)^{T} z(t)-\gamma^{2} w(t)^{T} w(t)$,

$$
\begin{aligned}
& 0=2\left[x(t)^{T} P_{2}^{T}+\dot{x}(t)^{T} P_{3}^{T}\right] \\
& {\left[A_{0} x(t)+B w(t)+\sum_{i=1}^{n} A_{i} x\left(t-h_{1}\right)-\sum_{i=1}^{n} A_{i} v_{1 i}-\dot{x}(t)\right]} \\
& 0=2\left[x(t)^{T} Y_{1}^{T}+\dot{x}(t)^{T} Y_{2}^{T}\right] \\
& {\left[n x\left(t-h_{2}\right)+\sum_{i=1}^{n} v_{1 i}+\sum_{i=1}^{n} v_{2 i}-n x\left(t-h_{1}\right)\right]}
\end{aligned}
$$

Setting,

$$
\begin{aligned}
\eta(t)= & \operatorname{col}\left\{x(t), \dot{x}(t), x\left(t-h_{1}\right), x\left(t-h_{2}\right),\right. \\
& \left.v_{11}, v_{12}, \ldots, v_{1 n}, v_{21}, v_{22}, \ldots, v_{2 n}, w(t)\right\}
\end{aligned}
$$

Finally, if the LMI in (8) is feasible, we obtain,

$$
\begin{aligned}
& \dot{V}(t, x(t), \dot{x}(t))+z(t)^{T} z(t)-\gamma^{2} w(t)^{T} w(t) \\
& \leq \eta(t)^{T} \Gamma^{2} \eta(t)<0
\end{aligned}
$$

\section{Problem Statement}

Own proxy control scheme for the bilateral teleoperation system is shown in Figure 1. 
- $F_{m}(t)$ and $F_{s}(t)$ are the actuated inputs of the master and the slave.

- $F_{h}(t)$ and $F_{e}(t)$ are the effects of the human operator and environment on the system, $\hat{F}_{h}(t)$ and $\hat{F}_{e}(t)$ are the estimations of these two forces, which can be obtained by adding the perturbation observers in reality (due to the space limitation, the perturbation observer will not be introduced in the paper).

- $\tau_{1}(t)$ (from the master to the slave) and $\tau_{2}(t)$ (from the slave to the master) are the time-varying delays, which are modeled in [13].

- $\dot{\theta}_{m}(t) / \theta_{m}(t), \dot{\theta}_{p}(t) / \theta_{p}(t)$ and $\dot{\theta}_{s}(t) / \theta_{s}(t)$ are the velocities/positions of the master, the proxy of master and the slave.

The controller $C$ and the proxy of master will be designed in sequential steps in next section. In particular, the proxy of master is like a remote observer of the master, which is used in the slave side to reduce the impact of the time-varying delays.

According to the novel scheme, The following assumptions are made,

1. The master and the slave are linear dynamical systems.

2. The communication delays are bounded, $\tau_{1}(t)$, $\tau_{2}(t) \in\left[h_{1}, h_{2}\right]$.

3. The data packet exchanged between the master, the proxy of master and the slave are time-stamped, so the master, the proxy of master and the slave clock are synchronized [13].

4. The master, the proxy and the slave systems have a local controller ensuring the marginal stability (only the speed stability), $K_{m}^{0}$ and $K_{s}^{0}$.

The objective of the paper is to design the proxy of master and the controller $C$ in Figure 1 to ensure the stability of the whole system and the position tracking between the master and the slave under time-varying delays. Besides, because of the characteristic of the control scheme, the force tracking, $F_{m}(t)=\hat{F}_{e}\left(t-\tau_{2}(t)\right)$, is realized, if the stability of the whole system is verified. The master is given by following model,

$$
\begin{aligned}
\left(\Sigma_{m}\right) \quad \dot{x}_{m}(t) & =\left(A_{m}-B_{m} K_{m}^{0}\right) x_{m}(t) \\
& +B_{m}\left(F_{m}(t)+F_{h}(t)\right)
\end{aligned}
$$

Where, $x_{m}(t)=\dot{\theta}_{m}(t)$ is the state of the master, $K_{m}^{0}$ is the local partial state feedback, it is supposed to be known as the assumption 4 above.

Figure 2 shows the scheme of the proxy of master, the block diagram 'Proxy' represents the proxy model, which is exactly same with the master. $L$ is the gain that will be designed, $\hat{\tau}_{1}(t)$ is the estimated network delay between the master and proxy. As the assumption 3 above, the delay can be measured at the proxy and slave side, $\hat{\tau}_{1}(t)=$ $\tau_{1}(t)$. The model of the proxy is as follow,

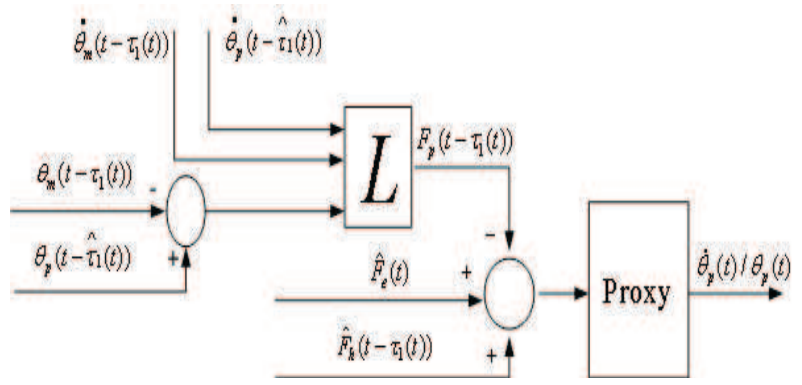

Figure 2. Proxy of master.

$$
\begin{aligned}
\left(\Sigma_{p}\right) \quad \dot{x}_{p}(t) & =\left(A_{m}-B_{m} K_{m}^{0}\right) x_{p}(t)-B_{m} F_{p}\left(t-\tau_{1}(t)\right) \\
& +B_{m}\left(\hat{F}_{e}(t)+\hat{F}_{h}\left(t-\tau_{1}(t)\right)\right)
\end{aligned}
$$

As the master, $x_{p}(t)=\dot{\theta}_{p}(t)$, and the gain $L$ is used to synchronize the position between the master and the proxy of master,

$$
\begin{aligned}
F_{p}\left(t-\tau_{1}(t)\right) & =L\left(\begin{array}{c}
\dot{\theta}_{p}\left(t-\hat{\tau}_{1}(t)\right) \\
\dot{\theta}_{m}\left(t-\tau_{1}(t)\right) \\
\theta_{p}\left(t-\hat{\tau}_{1}(t)\right)-\theta_{m}\left(t-\tau_{1}(t)\right)
\end{array}\right) \\
L & =\left(\begin{array}{lll}
L_{1} & L_{2} & L_{3}
\end{array}\right)
\end{aligned}
$$

Next, $K$ is the gain of the controller $C$,

$$
\begin{gathered}
F_{s}(t)=K\left(\begin{array}{c}
\dot{\theta}_{s}(t) \\
\dot{\theta}_{p}(t) \\
\theta_{s}(t)-\theta_{p}(t)
\end{array}\right) \\
K=\left(\begin{array}{lll}
K_{1} & K_{2} & K_{3}
\end{array}\right)
\end{gathered}
$$

Finally, the model of the slave is given,

$$
\begin{aligned}
\left(\Sigma_{s}\right) \quad \dot{x}_{s}(t) & =\left(A_{s}-B_{s} K_{s}^{0}\right) x_{s}(t)-B_{s} F_{s}(t) \\
& +B_{s} F_{e}(t)
\end{aligned}
$$

Where, $x_{s}(t)=\dot{\theta}_{s}(t)$.

\section{Main Results}

The objective of this section is to provide a control design for the proxy of master and the controller $C$, to achieve the stability of the whole system with guaranteed performance, the position/force tracking.

\subsection{The design of proxy of master}

Firstly, we will design the proxy of master by Lyapunov-Krasovskii functional, $H_{\infty}$ control and LMI. Taking into the master and the proxy, we describe the two systems as the stabilization of the following linear system,

$\left(\Sigma_{m p}\right)\left\{\begin{aligned} \dot{x}_{m p}(t) & =A_{m p}^{0} x_{m p}(t)+A_{m p}^{1} x_{m p}\left(t-\tau_{1}(t)\right) \\ & +B_{m p} w_{m p}(t) \\ z_{m p}(t) & =C_{m p} x_{m p}(t)\end{aligned}\right.$ 
Where,

$$
\begin{aligned}
& x_{m p}(t)=\left(\begin{array}{c}
\dot{\theta}_{p}(t) \\
\dot{\theta}_{m}(t) \\
\theta_{p}(t)-\theta_{m}(t)
\end{array}\right) \\
& w_{m p}(t)=\left(\begin{array}{c}
\hat{F}_{e}(t)+\hat{F}_{h}\left(t-\tau_{1}(t)\right) \\
F_{m}(t)+F_{h}(t)
\end{array}\right) \\
& z_{m p}(t)=\left(\theta_{p}(t)-\theta_{m}(t)\right)
\end{aligned}
$$

So,

$$
\begin{aligned}
A_{m p}^{0} & =\left(\begin{array}{ccc}
A_{m}-B_{m} K_{m}^{0} & 0 & 0 \\
0 & A_{m}-B_{m} K_{m}^{0} & 0 \\
1 & -1 & 0
\end{array}\right) \\
A_{m p}^{1} & =\left(\begin{array}{ccc}
-B_{m} L_{1} & -B_{m} L_{2} & -B_{m} L_{3} \\
0 & 0 & 0 \\
0 & 0 & 0
\end{array}\right) \\
B_{m p} & =\left(\begin{array}{cc}
B_{m} & 0 \\
0 & B_{m} \\
0 & 0
\end{array}\right)=\left(\begin{array}{ll}
B_{m p}^{1} & B_{m p}^{2}
\end{array}\right) \\
C_{m p} & =\left(\begin{array}{lll}
0 & 0 & 1
\end{array}\right)
\end{aligned}
$$

For applying LMI to the system above, we have made the transformation of the system term $A_{m p}^{1} x_{m p}\left(t-\tau_{1}(t)\right)$ as follow,

$$
A_{m p}^{1} x_{m p}\left(t-\tau_{1}(t)\right)=-B_{m p}^{1} L x_{m p}\left(t-\tau_{1}(t)\right)
$$

Then, the following stability theorem is obtained,

Theorem 3 Suppose there exists matrices $P>0, R>0$, $S>0, S_{a}>0, R_{a 1}>0, P_{2}, Y_{1}, Y_{2}, M$, and positive scales $\gamma$ and $\xi$, such that the condition (26) with the notation (27) is feasible, the system (21) is asymptotically stable and $J(w)<0$ for time-varying delays $\tau_{1}(t) \in\left[h_{1}, h_{2}\right]$. The control gain of the proxy is given by,

$$
L=M P_{2}^{-1}
$$

Proof. We substitute the system (21) into the theorem 2, and the proof can be found in [6].

Remark 1 The $H_{\infty}$ control design objective is to minimize the norm of the closed-loop mapping $w_{m p}(t) \rightarrow$ $z_{m p}(t)$. More precisely, we look for a minimum characterization of levels $\gamma$, which is defined as $\gamma_{\text {min }}^{L}$, therefore, the bound $\sup _{w_{m p}}\left(\left\|z_{m p}(t)\right\|_{2} /\left\|w_{m p}(t)\right\|_{2}\right)<\gamma_{m i n}^{L}$ is achievable in the closed-loop system. Therefore, by theorem 3 , we can minimize the deviation of position $z_{m p}(t)$, in the condition of exogenous disturbance input $w_{m p}(t)$, further achieve some minimal level of synchronization, as the position tracking. Besides, the performance of synchronization is proportional to the magnitude of $\gamma_{m i n}^{L}$, the smaller $\gamma_{\text {min }}^{L}$ produces the better performance of system [19].

\subsection{The design of the controller $C$}

The position tracking between the master and the proxy of master has been achieved. In this subsection, the position tracking between the proxy of master and the slave is proved by designing the controller $C$. The model of the system containing the proxy of master, the controller $C$ and the slave, is given as follow,

$$
\left(\Sigma_{p s}\right)\left\{\begin{aligned}
\dot{x}_{p s}(t) & =A_{p s} x_{p s}(t)+B_{p s} w_{p s}(t) \\
z_{p s}(t) & =C_{p s} x_{p s}(t)
\end{aligned}\right.
$$

Similarly,

$$
x_{p s}(t)=\left(\begin{array}{c}
\dot{\theta}_{s}(t) \\
\dot{\theta}_{p}(t) \\
\theta_{s}(t)-\theta_{p}(t)
\end{array}\right), \quad z_{p s}(t)=\left(\theta_{s}(t)-\theta_{p}(t)\right)
$$

Particularly, the input of the proxy, $F_{p}\left(t-\tau_{1}(t)\right)$, is considered as the exogenous disturbance signal,

$$
w_{p s}(t)=\left(\begin{array}{c}
F_{e}(t) \\
\hat{F}_{e}(t)+\hat{F}_{h}\left(t-\tau_{1}(t)\right)-F_{p}\left(t-\tau_{1}(t)\right)
\end{array}\right)
$$

So,

$$
\begin{aligned}
A_{p s} & =\left(\begin{array}{ccc}
A_{s}-B_{s} K_{s}^{0}-B_{s} K_{1} & -B_{s} K_{2} & -B_{s} K_{3} \\
0 & A_{m}-B_{m} K_{m}^{0} & 0 \\
1 & -1 & 0
\end{array}\right) \\
B_{p s} & =\left(\begin{array}{cc}
B_{s} & 0 \\
0 & B_{m} \\
0 & 0
\end{array}\right)=\left(\begin{array}{ll}
B_{p s}^{1} & B_{p s}^{2}
\end{array}\right), \quad C_{p s}=\left(\begin{array}{lll}
0 & 0 & 1
\end{array}\right)
\end{aligned}
$$

The controller gain $K$ should be calculated by LMI, but with the term $A_{p s}$, this is difficult to apply LMI, so the transformation is made,

$$
\begin{aligned}
A_{p s} x_{p s}(t) & =A_{p s}^{0} x_{p s}(t)+A_{p s}^{1} x_{p s}(t) \\
& =\left(\begin{array}{ccc}
A_{s}-B_{s} K_{s}^{0} & 0 & 0 \\
0 & A_{m}-B_{m} K_{m}^{0} & 0 \\
1 & -1 & 0
\end{array}\right) x_{p s}(t) \\
& +\left(\begin{array}{ccc}
-B_{s} K_{1} & -B_{s} K_{2} & -B_{s} K_{3} \\
0 & 0 & 0 \\
0 & 0 & 0
\end{array}\right) x_{p s}(t) \\
& =A_{p s}^{0} x_{p s}(t)-B_{p s}^{1} K x_{p s}(t)
\end{aligned}
$$

Then, we obtain the following theorem,

Theorem 4 Suppose there exists matrices $P>0, P_{2}, W$, and positive scales $\gamma$ and $\xi$, such that the condition (34) with the notation (35) is feasible, the system (28) is asymptotically stable and $J(w)<0$. The control gain of the controller $C$ is given by,

$$
K=W P_{2}^{-1}
$$




$$
\begin{aligned}
& \Gamma^{3}=\left(\begin{array}{cccccccc}
\Gamma_{11}^{3} & \Gamma_{12}^{3} & R-B_{m p}^{1} M-Y_{1}^{T} & Y_{1}^{T} & Y_{1}^{T}+B_{m p}^{1} M & Y_{1}^{T} & B_{m p} & P_{2}^{T} C_{m p}^{T} \\
* & \Gamma_{22}^{3} & -\xi B_{m p}^{1} M-Y_{2}^{T} & Y_{2}^{T} & Y_{2}^{T}+\xi B_{m p}^{1} M & Y_{2}^{T} & -\xi B_{m p} & 0 \\
* & * & -S-R & 0 & 0 & 0 & 0 & 0 \\
* & * & * & -S_{a} & 0 & 0 & 0 & 0 \\
* & * & * & * & -R_{a 1} & 0 & 0 & 0 \\
* & * & * & * & * & -R_{a 1} & 0 & 0 \\
* & * & * & * & * & * & -\gamma^{2} I & 0 \\
* & * & * & * & * & * & * & -I
\end{array}\right)<0 \\
& \Gamma_{11}^{3}=S+S_{a}-R+P_{2}^{T} A_{m p}^{T}+A_{m p} P_{2}, \quad \Gamma_{12}^{3}=P-P_{2}+\xi P_{2}^{T} A_{m p}^{T}, \quad \Gamma_{22}^{3}=-\xi P_{2}-\xi P_{2}^{T}+h_{1}^{2} R+\left(h_{2}-h_{1}\right)^{2} R_{a 1}
\end{aligned}
$$

$$
\begin{aligned}
\Gamma^{4}=\left(\begin{array}{cccc}
\Gamma_{11}^{4} & \Gamma_{12}^{4} & B_{p s} & P_{2}^{T} C_{p s}^{T} \\
* & \Gamma_{22}^{4} & \xi B_{p s} & 0 \\
* & * & -\gamma^{2} I & 0 \\
* & * & * & -I
\end{array}\right)<0 \\
\Gamma_{11}^{4}=P_{2}^{T} A_{p s}^{0}{ }^{T}+A_{p s}^{0} P_{2}-B_{p s}^{1} W-W^{T} B_{p s}^{1^{T}} \\
\Gamma_{12}^{4}=P-P_{2}+\xi P_{2} A_{p s}^{0}-\xi W^{T} B_{p s}^{1}{ }^{T} \\
\Gamma_{22}^{4}=-\xi P_{2}-\xi P_{2}^{T}
\end{aligned}
$$

Proof. Theorem 4 is an extended application of the theorem 1 , the proof of $K$ is similar to the theorem 3 .

Remark 2 Theorem 4 has achieved the position tracking between the proxy of master and the slave with the minimum $\gamma_{\text {min }}^{K}\left(\sup _{w_{p s}}\left(\left\|z_{p s}(t)\right\|_{2} /\left\|w_{p s}(t)\right\|_{2}\right)<\gamma_{\text {min }}^{K}\right)$. With the results of the subsections above, the position tracking of the master and the slave has been achieved.

\subsection{The global stability of the system}

Based on the position tracking, finally, the objective is to ensure the global stability of the system. In this context, the force tracking from the slave to the master can be realized on the basis of the characteristic of the control scheme.

The whole system is described,

$$
\left(\Sigma_{4}\right)\left\{\begin{aligned}
\dot{x}(t) & =A_{0} x(t)+A_{1} x\left(t-\tau_{1}(t)\right)+B w(t) \\
z(t) & =C x(t)
\end{aligned}\right.
$$

Where,

$$
\begin{aligned}
x(t)= & \left(\begin{array}{c}
\dot{\theta}_{s}(t) \\
\dot{\theta}_{p}(t) \\
\dot{\theta}_{m}(t) \\
\theta_{s}(t)-\theta_{p}(t) \\
\theta_{p}(t)-\theta_{m}(t)
\end{array}\right) \\
w(t)= & \left(\begin{array}{c}
F_{e}(t) \\
\hat{F}_{e}(t)+\hat{F}_{h}\left(t-\tau_{1}(t)\right) \\
F_{m}(t)+F_{h}(t)
\end{array}\right) \\
z(t)= & \left(\begin{array}{c}
\theta_{s}(t)-\theta_{p}(t) \\
\theta_{p}(t)-\theta_{m}(t)
\end{array}\right)
\end{aligned}
$$

So, we can get,

$$
\begin{aligned}
& A_{0}=\left(\begin{array}{ccccc}
A_{0}(1,1) & -B_{s} K_{2} & 0 & -B_{s} K_{3} & 0 \\
0 & A_{0}(2,2) & 0 & 0 & 0 \\
0 & 0 & A_{0}(3,3) & 0 & 0 \\
1 & -1 & 0 & 0 & 0 \\
0 & 1 & -1 & 0 & 0
\end{array}\right) \\
& A_{0}(1,1)=A_{s}-B_{s} K_{s}^{0}-B_{s} K_{1} \\
& A_{0}(2,2)=A_{m}-B_{m} K_{m}^{0}, \quad A_{0}(3,3)=A_{m}-B_{m} K_{m}^{0} \\
& A_{1}=\left(\begin{array}{ccccc}
0 & 0 & 0 & 0 & 0 \\
0 & -B_{m} L_{1} & -B_{m} L_{2} & 0 & -B_{m} L_{3} \\
0 & 0 & 0 & 0 & 0 \\
0 & 0 & 0 & 0 & 0 \\
0 & 0 & 0 & 0 & 0
\end{array}\right) \\
& B=\left(\begin{array}{ccc}
B_{s} & 0 & 0 \\
0 & B_{m} & 0 \\
0 & 0 & B_{m} \\
0 & 0 & 0 \\
0 & 0 & 0
\end{array}\right), \quad C=\left(\begin{array}{ccccc}
0 & 0 & 0 & 1 & 0 \\
0 & 0 & 0 & 0 & 1
\end{array}\right)
\end{aligned}
$$

By theorem 2, we can verify the global stability of the system. That is to say, with the force tracking, $F_{m}(t)=$ $\hat{F}_{e}\left(t-\tau_{2}(t)\right)$, the system is globally stable under the $H_{\infty}$ constraint.

Remark 3 The global design of $L$ and $K$ with the terms $A_{0}$ and $A_{1}$ is impossible, because the $L$ and $K$ in (38) can not be calculated in the form of LMI, which normally requires the transformation as (24), (32).

\section{Simulation}

To evaluate the performance of the proposed approach, different working conditions have been simulated. The maximum amplitude and sampling time of time-varying delays are $0.2 s$ (greater amplitude of delays can also be handled) and $0.001 s$, which satisfy most network-based applications of teleoperation system, as internet-based teleoperation system. Notice that, the time-varying delays in two channels are asymmetric.

In order to simplify the system simulation, the master, the proxy of master and the slave models can be described as $m_{m} / s, m_{m} / s$ and $m_{s} / s$. And for verifying the posi- 
tion tracking, the effective endpoint mass is chosen differently, $m_{m}=1$ and $m_{s}=2 \mathrm{~kg}$. Besides, the poles of the master, the proxy and the slave are given as [-100.0], then, $K_{m}^{0}=100, K_{s}^{0}=50$.

The subsystems mentioned above $\left(A_{m p}^{0}, B_{m p}, A_{p s}^{0}\right.$ and $\left.B_{p s}\right)$ can be obtained. Then, according to the theorems above, the gains of the proxy of master and the controller $C, L$ and $K$, are obtained by YALMIP/MATLAB,

$$
\begin{aligned}
& L=\left(\begin{array}{lll}
-1.6296 & 0.3669 & 283.5530
\end{array}\right), \quad \gamma_{\min }^{L}=0.0083 \\
& K=\left(\begin{array}{lll}
-29.9635 & -3.6393 & 618.5365
\end{array}\right) \\
& \gamma_{\text {min }}^{K}=0.0075
\end{aligned}
$$

And, the global stability of the system $\left(A_{0}, A_{1}\right.$ and $\left.B\right)$ is verified with $\gamma_{\text {min }}^{g}=0.0054$.

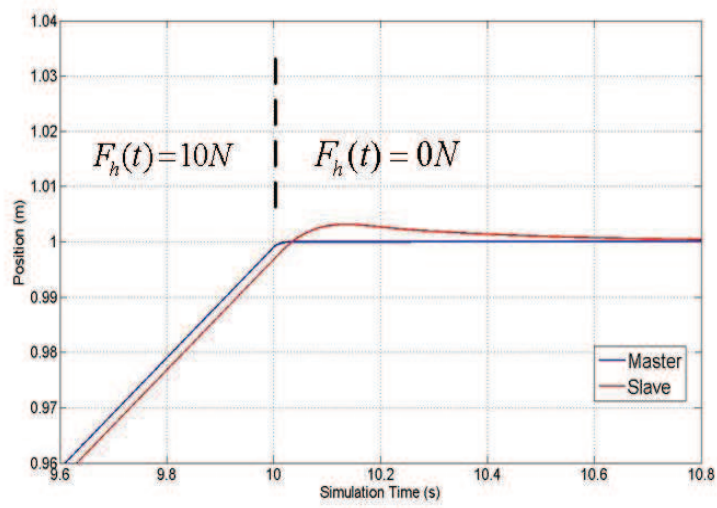

\section{Figure 3. Position response in abrupt changing motion.}

\subsection{Tracking in abrupt changing motion}

Because the free motion simulation can be represented by the abrupt changing motion, it is omitted here. For simulation purpose of the abrupt change, the human operator $\left(F_{h}(t)\right)$ is modeled as the pulse generator. Figure 3 shows the position tracking between the master and the slave. At the mutation point, good convergence between the master and the slave can be seen.

In this context, $F_{e}(t)=0$, so the actuated inputs of the master $F_{m}(t)=0$ in steady state.

\subsection{Tracking in wall contact motion}

We also performed simulation in wall contact motion, where the slave is driven to the hard wall with a stiffness of $K_{e}=30 \mathrm{kN} / \mathrm{m}$ located at the position $x=1.0 \mathrm{~m}$.

The upper part of Figure 4 shows the position tracking in [21], in which, the deviation of the position between the master and the slave is obvious. In the lower part of Figure 4 , the system shows a satisfactory position tracking with the method proposed in the paper under the same conditions.
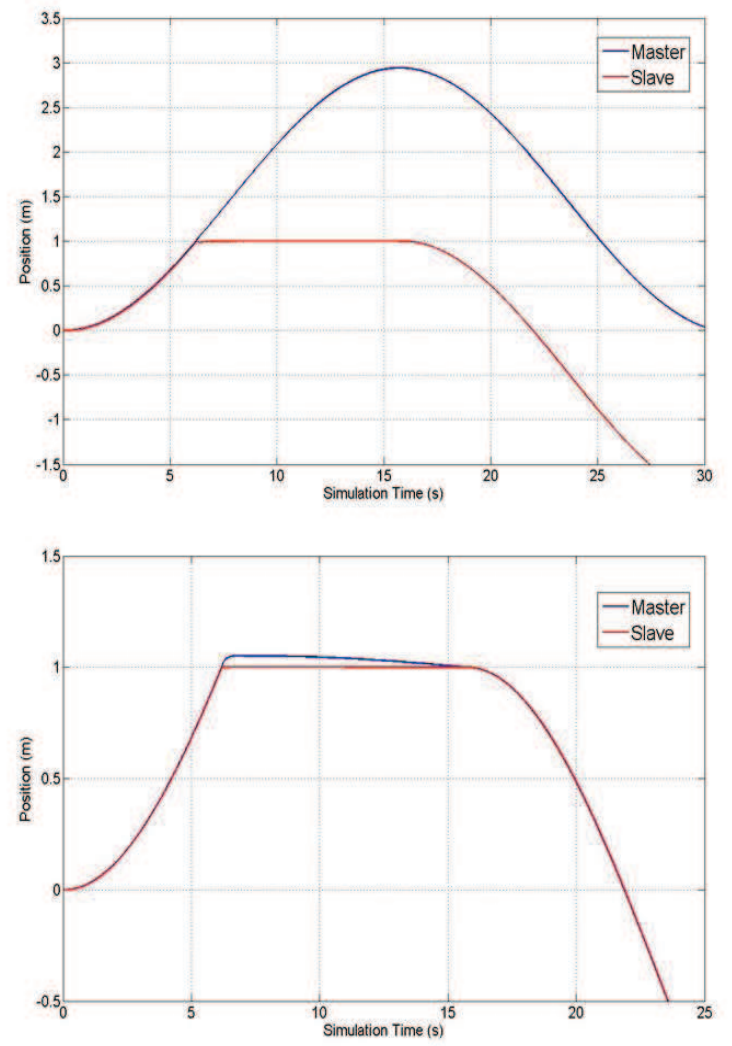

Figure 4. Position response in wall contact motion (upper: from [21]; lower: this paper)

Figure 5 shows the force tracking between the master and the slave, $F_{m}(t)$ and $\hat{F}_{e}(t)$. We added a simple perturbation observer in the scheme to obtain unmeasured $\hat{F}_{e}(t)$.

As the results of the simulation, the proposed method in the paper can greatly improve the performance of the system, the position tracking, and achieve the force tracking by the perturbation estimation and force feedback in steady state.

\section{Conclusions and Future Works}

In this paper, a new architecture and design for bilateral teleoperation has been proposed to obtain good position tracking, and then, comparable force tracking ability. It is based on the global stability analysis. The new configuration addresses asymmetric and time-varying delays, and builds upon the design of the proxy of master and the position error controller $C$. We use the theory of Lyapunov/Lyapunov-Krasovskii functional with $H_{\infty}$ control, which is given in the form of LMI. Moreover, the novel LMI-based architecture is a comprehensive summary of the Position-Position scheme and Position-Force scheme, and presents a more flexible tool for the control design of the bilateral teleoperation system.

Numerical simulations, achieved by YALMIP and SIMULINK, have confirmed the accuracy of analysis, and 


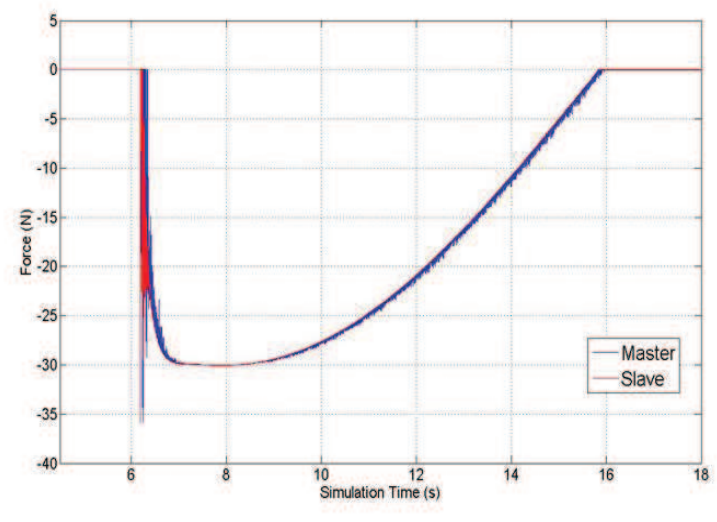

Figure 5. Force response in wall contact motion

proved that the teleoperation system, designed by our theory, can run in different workshop conditions.

The better estimation of the exogenous perturbation force will be entailed in the future work. Besides, the real implementations are being planned on an experimental testbench.

\section{References}

[1] R. W. Anderson and M. W. Spong. Bilateral control of teleoperators with time delay. IEEE Transactions on Automatic Control, 34(5):494-501, 1989.

[2] J. Cheong and S.-I. Niculescu. Robust motion synchronization control for interconnected systems with human interaction. In Proceedings of the 17th World Congress The International Federation of Automatic Control, pages 6347-6352, Seoul, Korea, July 2008.

[3] N. Chopra, M. W. Spong, R. Ortega, and N. E. Barabanov. On tracking performance in bilateral teleoperation. IEEE Transactions on Robotics, 22(4):861-866, 2006.

[4] E. Delgado and A. Barreiro. Stability of teleoperation systems for time-varying delays by Lyapunov-Krasovskii and frequencial techniques. In 35th Annual Conference of IEEE on Industrial Electronics (IECON), pages 17481753, Porto, Portugal, November 2009.

[5] E. Fridman. A new Lyapunov technique for robust control of systems with uncertain non-small delays. IMA Journal of Mathematical Control and Information, 23(2):165-179, 2006.

[6] E. Fridman and U. Shaked. New bounded real lemma representations for time-delay systems and their applications. IEEE Transactions on Automatic Control, 46(12):19731979, 2001.

[7] H. Fujita and T. Namerikawa. Delay-independent stabilization for teleoperation with time varying delay. In 2009 American Control Conference, pages 1221-1226, St. Louis, USA, June 2009.

[8] L. G. Garcia-Valdovinos, V. Parra-Vega, and M. A. Arteaga. Observer-based sliding mode impedance control of bilateral teleoperation under constant unknown time delay. Robotics and Autonomous Systems, 55(8):609-617, 2007.
[9] K. Gu, V. L. Kharitonov, and J. Chen. Stability of timedelay systems. Boston, Basel, Berlin, 2003.

[10] Y. He, M. Wu, J.-H. She, and G.-P. Liu. Parameterdependent Lyapunov functional for stability of time-delay systems with polytopic-type uncertainties. IEEE Transactions on Automatic Control, 49(5):828-832, 2002.

[11] Z.-L. Hou, Q. Wei, F. Li, and D.-P. Han. Design on a half closed-loop bilateral teleoperation system. In Proceedings of 29th Chinese Control Conference, pages 3432-3436, Beijing, China, July 2010.

[12] C.-C. Hua and X. P. Liu. Delay-dependent stability criteria of teleoperation systems with asymmetric time-varying delays. IEEE Transactions on Robotics, 26(5):925-932, 2010.

[13] W.-J. Jiang, A. Kruszewski, J.-P. Richard, and A. Toguyeni. A gain scheduling strategy for the control and estimation of a remote robot via internet. In 27th Chinese Control Conference, pages 793-799, Kunming, China, July 2008.

[14] K. Natori, T. Tsuji, K. Ohnishi, A. Hace, and K. Jezernik. Time-delay compensation by communication disturbance observer for bilateral teleoperation under timevarying delay. IEEE Transactions on Industrial Electronics, 57(3):1050-1062, 2010.

[15] G. Niemeyer and J.-J. E. Slotine. Stable adaptive teleoperation. IEEE Journal of Oceanic Engineering, 16(1):152$162,1991$.

[16] J.-P. Richard. Time delay systems: an overview of some recent advances and open problems. Automatica, 39(10): 1667-1694, 2003.

[17] J.-H. Ryu, C. Preusche, B. Hannaford, and G. Hirzinger. Time domain passivity control with reference energy following. IEEE Transactions on Control Systems Technology, 13(5):737-742, 2005.

[18] M. Shahbazi, H. A. Talebi, and M. J. Yazdanpanah. A control architecture for dual user teleoperation with unknown time delays: A sliding mode approach. In 2010 IEEE/ASME International Conference on Advanced Intelligent Mechatronics (AIM), pages 1221-1226, Montreal, Canada, July 2010.

[19] G. Tadmor. The standard $H_{\infty}$ problem in systems with a single input delay. IEEE Transactions on Automatic Control, 45(3):382-397, 2000.

[20] S.-Y. Xu, J. Lam, and Y. Zou. New results on delaydependent robust $H_{\infty}$ control for systems with timevarying delays. Automatica, 42(2):343-348, 2006.

[21] Y.-Q. Ye, Y.-J. Pan, and Y. Gupta. Time domain passivity control of teleoperation systems with random asymmetric time delays. In Proceedings of 48th IEEE Conference on Decision and Control, and 28th Chinese Control Conference, pages 7533-7538, Shanghai, China, December 2009.

[22] S. Zampieri. Trends in networked control systems. In Proceedings of the 17th World Congress The International Federation of Automatic Control, pages 2886-2894, Seoul, Korea, July 2008.

[23] B. Zhang, A. Kruszewski, and J.-P. Richard. A novel control scheme for teleoperation with guaranteed performance under time-varying delays. In 23th Chinese Control and Decision Conference, Accepted, Mianyang, China, May 2011.

[24] X.-M. Zhang and Q.-L. Han. Robust $H_{\infty}$ filtering for a class of uncertain linear systems with time-varying delay. Automatica, 44(1):157-166, 2008. 